\title{
Computing Environments, Interactive Graphics and Nonparametric Methods for Data Analysis
}

\author{
(Grant DE-FG06-5-ER25006 A003)
}

Final Report

\author{
John Alan McDonald \\ Finbarr O'Sullivan \\ Werner Stuetzle \\ Department of Statistics \\ University of Washington \\ Seattle, WA 98195
}

May 1, 1993

\section{DISCLAIMER}

This report was prepared as an account of work sponsored by an agency of the United States Neither the United States Government nor any agency thereof, nor any of their Government. Neither the Uny legal liability or responsiemployees, makes any warranty, express or impled, or ass information, apparatus, product, or bility for the accuracy, completeness, or usefulness of and infinge privately owned rights. Referprocess disclosed, or represents that its use would not infring, ence herein to any specific commercial product, process, or service by te its endorsement, recommanufacturer, or otherwise does not necessarily constitute or imply its endorsem thereof. The views mendation, or favoring by the United States Government or any agency therect those of the and opinions of authors expressed herein do not 


\section{Introduction}

This final report covers the funding period from July 15,1988 to July 15 , 1992. Sections 2-4 of the report summarize the research in the areas of computing environments, interactive graphics, and nonparametric methods, respectively. Section 5 contains a list of all publications prepared under the grant. Section 6 lists the Ph.D. theses that were partially or completely supported by the grant. Section 7 lists the invited lectures in which grant sponsored work was presented.

\section{Computing environments}

The Arizona system is intended to be a portable, public-domain collection of tools supporting scientific computing, quantitative graphics, and data analysis, implemented in Common Lisp and CLOS (the Common Lisp Object System).

The design of Arizona is motivated by our belief that an ideal system for scientific computing and data analysis should have:

- One language that can be used for both for line-by-line interaction or defining compiled procedures.

- Minimal overhead in adding new compiled procedures (or other definitions).

- A language that supports a wide variety of abstractions and the definition of new kinds of abstractions.

- Programming tools (editor, debugger, browsers, metering and monitoring lools).

- Automatic memory management (dynamic space allocation and garbage collection).

- Portability over many types of workstations and operating systems.

- A community of users and developers.

- Access to traditional Fortran scientific subroutine libraries or equivalents. 
- A representation of scientific data directly in the data structures of the language.

- Comprehensive numerical, graphical, and statistical functionality.

- Device independent static output graphics.

- Window based interactive graphics.

- Support for efficient and concurrent access to large databases.

- Documentation and tutorials, both paper and on-line.

One reason Arizona is based on Common Lisp is that the first nine points (through "access to Fortran") come for free with standard Common Lisp environments. The remaining six are the research aspects of Arizona.

Arizona is divided into a number of modules with limited interdependencies, to permit individual modules to stabilize and be "released" and used independently. The word "module" is used here informally. The definitions in a module will usually be in a package defined for that module and the source code for those definitions will usually be found in files in a single directory. A number of the modules in Arizona are described below. Current modules in alpha release are Arizona-Tools, Definitons, Announcements, Actors, Geometry, Slate, Chart, Graph, Browser, and an interface between Lisp and the Fortran optimization package NPSOL. Four other modules have seen some use but are likely to have more significant changes before any sort of release: Clay, Basic-Math, Probability, and Cactus.

\section{Arizona-Tools}

The Arizona-Tools module contains assorted general purpose utilities for Common Lisp programming, which don't have an obvious home in any of the other modules.

\section{Definitions}

The Definitions module provides the beginnings of a database for Common Lisp source code objects.

The primary purpose of Definitions is to make possible convenient runtime access to information available in Common Lisp source code, that is lost in the normal process of reading, evaluating, and/or compiling.

\section{Announcements}


The Announcements module provides a simple mechanism for maintaining dependencies between objects through message broadcasting. The basic idea is to allow an object (the announcer to notify other objects (the audience) of a change of state (or some other event) without the announcer having to know of which objects need to be notified of which events.

\section{Actors}

The Actors module is primarily intended as a mechanism for interpreting and distributing asynchronous (input) events in a multiprocessing Lisp environment.

An Actor is an object with a message queue; each actor handles its messages in its own, separate, thread of computation, at least conceptually in parallel with other actors and in parallel with any read-eval-print loops or other I/O processes that may exist.

\section{Geometry}

The Geometry module is intended to support common geometric calculations arising in graphics, numerical linear algebra, optimization, and scientific computing in general. The goal is to allow geometric computation using abstractions that directly represent high level mathematical concepts like affine spaces, vectors, and linear transformations, while retaining the level of performance provided by traditional scientific subroutine packages like Linpack.

\section{Slate}

Slate is a low level "uevice-independent" graphics package that has been ported to a number of Common Lisp platforms, window systems, and other graphics devices. It is intended to be used by developers of higher level scientific and statistical graphics systems rather than end users. Slate is something like a simplified version of CLX, ported to run on window systems and graphics devices other than X11.

\section{Chart}

Chart is a simple example of a (slightly) higher level graphics package built on top of Slate. It is a quick and dirty approximation to S style graphics. What we mean by "S style graphics" is output-only, line and point plots, with labels and tic marks, where locations are specified in an arbitrary $2 \mathrm{~d}$ world coordinate system and the scaling to screen coordinates is done more or less automatically. 


\section{Graph}

The Graph module provides a simple protocol for data structures used to represent graphs (networks), graph nodes, and graph edges, and a sample implementation of that protocol.

\section{Browser}

The Browser module provides a generic graph browser, implemented using the Clay and Graph modules and the graph layout methodology described below.

\section{Lisp-NPSOL Interface}

The NPSOL package provides a number of functions for calling the Fortran optimization package NPSOL from Common Lisp, using the foreign function interface provided with Franz Allegro Common Lisp.

\section{Clay}

Clay is the beginnings of a system designed to support drawing and interacting with $2 \mathrm{~d}$ (and $3 \mathrm{~d}$ ) pictures for visualizing scientific data, both the observational data that's the usual domain of statisiicians and the computed "data" arising in computer experiments and algorithm animation. It provides a toolkit of standard plot components and mechanisms for pasting components together to make it easy for the user to improvise new kinds of plots. All plot components obey a clearly defined protocol. This means that there is a consistent user interface and that users can reliably define new types of plot components that serve in the existing user interface.

\section{Basic Math}

The Basic Math module provides data structures and procedures implementing relatively low-level numerical analysis functionality. It is a building block for the Cactus module. Included in Basic Math are: machine constants, special functions (eg. beta, gamma) extended vector operations (analogous to the BLAS used in Linpack, evaluation and interpolation (eg. generic continued fractions) 1d numerical integration, and basic random number generators.

\section{Probability}

The Probability module supports inference and Monte Carlo simulation (including bootstrapping) in a unified framework through a protocol for Probability-Measure classes. Probability measure objects are responsible 
for generating samples from themselves, computing their quantiles, and computing the probabilities of appropriate sets, including tail probabilities. The defined probability measure classes includes the standard oneand higher-dimensional parametric densities and discrete distributions, and non-parametric measures, either resulting from density estimates or the empirical measure of a set. (It's worth noting that simple descriptive statistics like mean, median, etc., are generic functions in the probability measure protocol and are applied to data sets by viewing them as empirical distributions.)

\section{Cactus}

Cactus is a system for numerical linear algebra and optimization implemented in CLOS. Cactus is designed to closely model the abstractions used in a course on finite dimensional (vector) spaces. It provides representations for a variety of spaces, points (elements of some space), and mappings between spaces. The linear algebra core of Cactus deals with vector spaces, vectors, and linear transformations; more general spaces, points, and mappings are used are used to support constrained optimization at a similar level of abstraction.

The implementation of Cactus is, in part, an experiment to see how closely a program can follow natural mathematical abstractions, without losing the level of performance in large numerical problems provided by traditional Fortran subroutine libraries. To evaluate the results of this experiment, I have compared, in the design, implementation, and performance of Cactus on certain standard numerical linear algebra problems with Linpack, a classic high-quality Fortran package for solving systems of linear equations and related problems.

The result of the experiment was that the overhead in using a high level of abstraction is modest - the runtimes of Cactus versions of common matrix decompositions range from about the same as the corresponding Linapck routines to perhaps about $50 \%$ longer. At the same time, the object-oriented design of Cactus eases code reuse and customization in ways that are not possible in Fortran.

Publications: $1,3,22,23,34,35,36,37,39,40,41$.

Lectures: $9,11,12,15$. 


\section{Interactive graphics and visualization}

Our work in interactive graphics and visualization falls into four areas:

- Identification of abstractions

- Software architecture

- New kinds of displays and interaction techniques

- Declarative graph layout

\subsection{Identification of abstractions}

Researchers from areas as diverse as Computer Science, Statistics, and Cognitive Psychology have proposed a large and sometimes bewildering set of tools for visualizing large and complex collections of information. One of our goals has been to survey this work and to look for unifying principles. Extraction of such principles is important for several reasons:

- It greatly simplifies exposition.

- It allows construction of consistent and intuitive user interfaces.

- It suggests new combinations and extensions.

We have identified two unifying concepts, focusing and linking, that permeate much of the diverse visualization methodology.

To display complicated information, like a large program, an automobile and all its parts, or some multivariate statistical data, a common instinct is to draw a picture that is equally complicated, such as printing the program on the screen in a small font, rendering the automobile and its parts as transparent solids, or presenting the data as a tableau of Chernoff faces. Attempts at such dense encoding are seldom successful. It is usually more effective to construct a number of simple, easy to understand displays, each focused clearly on a particular aspect of the underlying data.

Focusing techniques may involve selecting subsets, dimension reduction, or some more general manipulation of the layout of information on the page or screen.

A consequence of focusing is that each view will only convey partial information about the data. We can compensate for this fact by displaying multiple 
views. Multiple views, however, should not be regarded in isolation. They need to be linked so that the information contained in individual views can be integrated into a coherent image of the data as a whole.

How views can be linked depends on whether they are displayed sequentially over time, or simultaneously.

The principal mechanism for linking views over time is through smooth change in the position of objects on the screen. Examples for this kind of linking are rotating three-dimensional point clouds and a higher dimensional generalization, the Grand Tour. More generally, any smooth animation can be considered as a set of linked multiple views, spread out over time..

Probably the most effective technique for linking simultaneous views is painting. Painting allows a user to interactively mark parts of a display with color or some other form of highlighting. The marking is automatically propagated to the other displays.

Publications: $4,11,12,47$.

Lectures: $1,16,23,25$.

\subsection{A software architecture for focusing and linking}

The basic building blocks of our architecture are:

\section{A database of subjects}

There is an object (a Subject) in the programming environment representing each abstract entity (eg., a multispectral image pixel) that the user might want to examine, select with the mouse, and manipulate.

Each Subject has associated with it a set of Attributes; an Attribute has an identity independent of any particular Subject. We can evaluate an Attribute for any Subject for which it is defined and we can change the value for some, if not all, Attributes. We can also define new Attributes for a given Subject at runtime.

Our programming model also presumes that Subjects can be organized into structured Collections, eg. lists, directed graphs, or dictionaries. We also presume that new types of Collections can be defined - if not by a random user, then at least by a programmer sophisticated enough to develop new kinds of plots.

Presentations in a Scene 
The Scene consists of a directed acyclic graph (usually a tree) of objects (Scene Nodes) representing geometric shapes (including text), in a two or three dimensional Scene Space. The Scene is rendered onto a display surface by standard computer graphics techniques. Some nodes in the Scene are visible representations or Presentations of some of the Subjects.

A Subject determines the position and appearance of its Presentations through an output Lens (see below). The hierarchical or graph structure of the Scene will often reflect a similar hierarchy in the Subjects; in other words, a Presentation of a Collection may have children that are Presentations of the elements of the Collection.

\section{Links}

There are three kinds of connections between Presentations and Subjects:

- Lenses for output filtering. A Lens maps from Subjects to be visualized into draw-able colored geometric shapes (including text) in the Scene space; it computes the position and appearance of a Presentation from the Attributes of its Subject.

- Input-Translators. An Input-Translator maps input events received by a Presentation to operations on its Subject.

- Consistency-Constraints. A Consistency-Constraint ensures that the Scenes always reflect the current state of the Subjects.

As a test of our programming model, we have implemented a very demanding variation on the notion of painting multiple views: multichannel image painting. "Paintir.o multiple views" means, in this context, that the user can interactively paint a representation of a pixel in any window, and all other representations of that pixel automatically take on the same color. For example, if the user sees a cluster in a scatterplot of channel space, it can be painted red to see where it falls in the image. Interesting objects in the image (eg. the brain or a known tumor in a medical image, a region with known land use classification in a satellite image, etc.) can be painted to see if they correspond to well defined clusters in channels space.

The key issue is squeezing out maximum performance, which is necessary for interactive response in even the smallest images. Our image painting implementation, based on the programming model described above, provides adequate interactive response with images up to size $1024 \times 1024 \times 6$ (Landsat TM) pixels. 
Publications: 4, 29, 30, 47.

Lectures: 1, 16, 23, 25.

\subsection{New kinds of displays and interaction techniques}

We have invented a a variety of new displays and interaction techniques.

\subsubsection{Odds plots}

The goal of scatterplot painting is to detect associations between variables shown in different plots. This requires visual estimation of relative densities, a difficult task especially in the presence of overstriking. The idea behind odds plots is to estimate relative densities algorithmically and display the result. Examples demonstrate the effectiveness of this approach.

\subsubsection{Variable resolution plots}

When drawing scatterplots, overstriking is a frequently occuring problem. It can result in an incorrect and misleading perception of the data density. If overstriking is serious, it is better to draw an agglomerative display, such as a two-dimensional histogram. The basic idea behind variable resolution plots is to calculate the amount of overstriking on the screen, and choose the appropriate display type accordingly. This means that the display type may change automatically when a plot is resized. Plots can be shrunk to a tiny size, and the two-dimensional histogram will still give a rough impression of the data density, while enlarging them will reveal more detail.

\subsubsection{Magnification lenses and logical zooming}

A magnification lense is a small rectangle that can be moved around on the screen under mouse control. The screen area under the lense is shown magnified in a separate window. The idea behing logical zooming is to replace simple geometric magnification by a more complicated process that also increases the type and amount of information presented. For example, an observation might be displayed in a scatterplot as a featureless dot. Applying a magnification lense might show the label of the observation, and further magnification might display additional attributes. Magnification lenses permit the inspection of details, while preserving context. 


\subsubsection{Automatic linking}

We have identified focusing and linking multiple views as basic concepts in visualization. Methods for automatic focusing, like principal component analysis and exploratory projection pursuit, have been available for some time. Automatic scatterplot linking works on the assumption that several views of the data have already been chosen, and the goal is to find associations between them. In the context of scatterplot painting we have developed various strategies for coloring observations in a way that makes associations apparent.

Publications: $26,45,46$.

Lectures: $6,7,8,18,19,20$.

\subsection{Declarative graph layout}

Automatic layout of graphs is problem that has a sizable literature. Roughly speaking, methods for layout can be divided into procedural and declarative (also known as constraint-busted).

By procedural layout, we mean simply that there is a procedure, which, given a graph, computes its layout. A typical layout procedure traverses the graph, assigning each node to an unoccupied site in a regular grid of allowed positions. The order of traversal and the method of site assignment are chosen in the hope of producing a "readable" layout: a minimal number of crossed edges, short (but not too short) edges, almost straight paths between important pairs of non-adjacent nodes, node positions whose y (or $x)$ coordinates are consistent with the partial ordering of a directed graph, and so on. There are often additional passes over the graph during which the nodes are re-arranged in an attempt to improve the layout.

Declarative layout could be thought of as a special case of procedural layout, where the procedure definition is based on three basic abstractions:

- a domain of possible layouts (for a given graph),

- a specification "language" that allows the user to express essential and desirable features of the layout, and

- a generic solver, that takes a graph and a specification, and returns an element of the domain that meets or well approximates the specification. 
For example, the domain might be all possible assignments of nodes to positions in a discrete lattice in the plane. The specification language might allow the user to ask to minimize some loss function (eg. the number of edge crossings) subject to constraints on the node positions (eg. no two nodes can occupy the same position in the lattice).

The distinction between procedural and declarative layout is, of course, very similar to the distinction between procedural and declarative programming languages. In particular, our notion of declarative layout is intentionally close to constraint programming languages.

The advantage of procedural layout is speed. A procedural method makes one or a few passes over the graph; the declarative example given above is an expensive integer programming problem, whose solution would require the equivalent of many iterations over the graph.

The advantage of the declarative approach is flexibility. In general, the only way for a user to modify the result of a procedural layout is to reprogram the procedure; because of the ad hoc nature of most layout procedures, it is difficult to know how to change the procedure to get the desired effect. The three abstractions of the declarative approach - the domain, the specification, and the solver - gives a user immediate access to a whole class of layouts. The key research issue is io design abstractions to provide a large and useful class of layouts, without giving up, too much in performance compared ad hoc procedural methods.

Our proposal for declarative layout is based on a physical analogy: Imagine a spring placed between each pair of nodes in the graph. Let the rest length of the spring be the desired distance between the nodes, eg., proportional to the length of the shortest path in the graph connecting the two nodes. Then minimize the energy.

The dcmain of the layout is the space of node positions, $\oplus^{n} \Re^{2}$, that is, the $\mathrm{n}$-fold direct sum of the plane. The specification language is the set of rest lengths (and spring constants). The solver is whatever minimization routine is used.

Our extension to the spring model is to minimize the energy subject to affine inequality (and equality) constraints on the node positions. For our solver, we are using standard packages for constrained minimization, in particular MINOS and NPSOL. The possibility of affine constraints gives us a much richer specification language, making it easy, for example, to preserve the directedness of a graph in the vertical or horizontal ordering of the nodes, or to adjust a layout for the size and shape of the window in which the graph 
is being displayed.

The use of modern optimization methods, as represented by MINOS and NPSOL, gives us still greater flexibility. We can experiment with other energy functions than the spring model and eventually provide users with more general ways to specify the energy than the rest lengths and spring constants. For example, we are considering energy functions that depend on the relationships of pairs of edges as well on pairs of nodes - with the goal of minimizing or at least controlling edge crossings.

Publications: 23, 27, 42 .

Lectures: $10,11,12,15$.

\section{Nonparametric methods}

Our research in nonparametric methods falls mainly in four areas:

1. Surface reconstruction

2. Multi-dimensional function estimation

3. System identification

4. Image analysis

\subsection{Surface reconstruction}

The general goal of our reserach in this area is the development of methods for reconstruction of curves and surfaces from partial information. 'The more specific problem we have addressed so far is that of reconstructing a two-dimensional manifold $S$ from an unorganized set of points $\mathbf{x}_{1}, \ldots, \mathbf{x}_{n}$ assumed to have been sampled densely and somewhat uniformly, with moderate noise, from $S$. Neither the topology nor the geometry of $S$ are assumed to be known in advance; they both have to be inferred from the data. This problem occurs in diverse scientific and engineering applications, such as reconstruction of three-dimensional objects from multi-view range finder data, reconstruction of biological shapes from multiple sets of contours, and interactive surface sketching.

We have invented a three phase procedure for the surface reconstruction problem. 
The goal of phase one is to estimate the topology of the unknown manifold $S$ and produces a crude approximation to its geometry. Our method for phase one consists of two major steps. In the first step we define a function $f$ which, for any point $\mathbf{x}$ close to $S$, approximates the signed distance between $\mathbf{x}$ and $S$. In the second step we use a contouring algcrithm (a version of marching cubes) to extract the zero-set of $f$. This zero-set, a simplicial surface (or mesh) $M_{0}$, is our initial, crude estimate for $S$. An estimate of the topological type of $S$ can be easily obtained from $M_{0}$ by counting the vertices, edges, faces, and boundary components.

The goal of phase two is to improve the fit of the mesh $M_{0}$ to the data and reduce the number of faces, while leaving the topological type unchanged.To solve this problem we define a energy function that captures the competing desires of tight geometric fit and compact representation. The trade off between geometric fit and compact representation is controlled via a user-selectable parameter $c_{r e p}$. A large value of $c_{\text {.ep }}$ indicates that compact representations are to be strongly preferred over dense ones, usually at the expense of degrading the fit.

Our algorithm for minimizing the energy function uses the mesh $M_{0}$ as a starting point for a non-linear optimization process. During the optimization we vary the number of vertices, their positions, and their connectivity. Although we can give no guarantee of producing a global minimum, the local minima produced by our method are generally quite good.

Although we were originally led to consider the mesh optimization problem by our research into surface reconstruction, the algorithm we have developed can also be applied to the problem of mesh simplification. Mesh simplification refers to the problem of reducing the number of faces in a dense mesh while minimally perturbing the shape. Mesh optimization can be used to solve this problem as follows: sample data points $X$ from the initial mesh and use the initial mesh as the starting point $M_{0}$ of the mesh optimization procedure.

The goal of phase three is to replace the mesh generated during phase two with a spline surface. The spline surface should be generally smooth, but should be allowed to have discontinuous derivatives wherever the data suggest presence of sharp edges in the target surface. Methods for phase three are currently under investigation.

Publications: $13,14,28,38,44$. 
Lectures: $3,4,5,24,30,31$.

\subsection{Multi-dimensional function estimation}

The goal of function estimation is the recovery of a function $f$ from a collection of possibly noisy observations. We have been working on nonparametric methods, making only few and very general assumptions about $f$. Nonparametric function estimation is an ill-posed problem, and is typically regularized by imposing a smoothness constraint on the estimate. The case where the domain of $f$ is univariate and its value is euclidean has been extensively studied, and good methods, such as spline smoothing and kernel smoothing, have existed for some time. Our contribution has been in three areas:

- Fast methods for multi-dimensional domains

- Methods for dealing with censored data.

- Mathematical analysis of nonparametric estimation procedures.

Publications: $6,7,9,18,19,33$

Lectures: $13,26$.

\subsection{System Identification}

Here the interest was in the estimation of parameters in system identifcation problems. Initially the work concentrated on problems arising in reservoir modeling; more recently there has been an increasing emphasis on examples arising in medical imaging. A thesis supported by the grant (K. Anderson) considers a non-linear deconvolution problem arising in the analysis of episodic hormone data whose structure is closely related to the deconvolution problem arising in reflection seismology.

Publications: $8,10,16,48$.

Lectures: $14,27,28$. 


\subsection{Image Analysis}

Work in this area has been motivated mainly by applications in emission tomography.

Publications: 5, 6, 16, 17, 20, 49, 50 .

Lectures: $21,22,27,28,29$. 


\section{Publications}

\section{Refereed Publications}

[1] J.A. McDonald and J.O. Pedersen. Computing environments for data analysis III: Programming environments. SIAM Journal on Scientific and Statistical Computing, Vol. 9(2), pp. 380-400, 1988.

[2] T. Hastie and W. Stuetzle. Principal curves. Journal of the American Statistical Association, Vol. 84, pp. 502-516, 1989.

[3] J.A. McDonald. Object-oriented programming for linear algebra. SIGPLAN Notices, Vol. 24 (10), pp. 175-184, 1989.

[4] J.A. McDonald, W. Stuetzle, and A. Buja. Painting Multiple Views of Complex Objects. SIGPLAN Notices, Vol. 25 (10), pp. 245-257, 1989.

[5] F. O'Sullivan, C. Wash, M. Stewart, and C. Motel. Rainfall estimation from infrared and visual GOES statellite imagery. Journal of Applied Meteorology, Vol. 29, pp. 209-223, 1990.

[6] F. O'Sullivan. Two-dimensional Laplacian smoothing by iterative methods with an application to image restoration. Journal of the American Statistical Association, Vol. 85, pp. 213-219, 1990.

[7] D.D. Cox and F. O'Sullivan. Asymptotic analysis of penalized likelihood and related estimators. Annals of Statistics, Vol. 18(4), pp. 1676-1695, 1990.

[8] F. O'Sullivan. Constrained non-linear least squares regularization with application to the estimation of functional parameters in elliptic partial differential equations. SIAM Journal on Numerical Analysis, Vol. 12, pp. 1635-1649, 1990.

[9] F. O'Sullivan. Discrete Laplacian smoothing by Fourier methods. Journal of the American Statistical Association, Vol. 86, pp. 634-642, 1991.

[10] F. O'Sullivan. Sensitivity Analysis for regularized estimation in some system identification problems. SIAM Journal on Scientific and Statistical Computing, Vol. 12, pp. 1266-1283, 1991.

[11] A. Buja, J.A. McDonald, J. Michalak, and W. Stuetzle. Interactive data visualization using focusing and linking. In: Proceedings of Visualization'91, San Diego, 1991.

[12] A. Buja, J.A. McDonald, J. Michalak, W. Stuetzle, and S. Willis. Visualization of complex data. 17 minute video tape. In video proceedings of Visualization'91, San Diego, 1991. 
[13] H.Hoppe, T. DeRose, T. Duchamp, J.A. McDonald, and W. Stuetzle. Surface reconstruction from unorganized points. Computer Graphics, Vol 26, (SIGGRAPH92 Proceedings), pp. 71-78, 1992.

[14] H.Hoppe, T. DeRose, T. Duchamp, J.A. McDonald, and W. Stuetzle. Mesh optimization. SIGGRAPH93 Proceedings (to appear), 1993.

[15] D. Donnell, A. Buja, and W. Stuetzle. Additive principal components. Annals of Statistics (to appear), 1993.

[16] F. O'Sullivan. Imaging radiotracer model parameters in PET: A mixture analysis approach. IEEE Transactions on Medical Imaging (to appear), 1993.

[17] Y. Pawitan and F. O'Sullivan. Data dependent bandwidth selection for emission computed tomography reconstruction. IEEE Transactions on Medical Imaging (to appear), 1993.

[18] F. O'Sullivan. Non-parametric Estimation in the Cox Proportional Hazards Model. Annals of Statistics (to appear), 1993.

[19] F. O'Sullivan and Y. Pawitan. Tomographic density estimation. Journal of the Royal Statistical Society, Series B, (to appear), 1993.

[20] F. O'Sullivan. Mixture estimation with multichannel image data. Journal of the American Statistical Association (to appear), 1993.

\section{Unrefereed Invited Publications}

[21] J.A. McDonald. Interactive graphics for data analysis. In W.S. Cleveland and M.E. McGill, editors, Dynamic graphics for statistics, pp. 247-275. Wadsworth and Brooks/Cole, Belmont, CA, 1988.

[22] J.A. McDonald. An outline of Arizona: a portable Lisp-based system for data analysis. In Proceedings of: Computer Science and Statistics, 20th Symposium on the Interface, Reston, Va., April 1988. (Also Technical Report 131, Dept. of Statistics, University of Washington)

[23] J.A. McDonald and J.O. Pedersen. A case study in statistical computing in a lisp environment: Geometric abstractions for constrained optimization of layouts. In Proceedings of the 1989 Joint Statistical Meetings, Statistical Computing Section, 1989.

[24] W. Stuetzle. Discussion of the article OMEGA - Online multivariate exploratory graphical analysis: routine search for structure by C. Weihs and H. Schmidli. Statistical Science, pp. 217-218, 1990. 
[25] F. O'Sullivan. Discussion of the MARS paper by I.H. Friedman. Annals of Statistics, pp. 1676-1695, 1990.

[26] W. Stuetzle. Odds Plots: A graphical aid for finding associations between views of a data set. In Andreas Buja and Paul Tukey, editors, Computing and graphics in statistics, volume IMA 36, Springer-Verlag, 1991.

[27] J.A. McDonald and J.O. Pedersen. Geometric abstractions for constrained optimization of layouts. In Andreas Buja and Paul Tukey, editors, Computing and Graphics in Statistics, volume IMA 36. Springer-Verlag, 1991.

[28] H. Hoppe, T.DeRose, T. Duchamp, J.A. McDonald, and W. Stuetzle. Fitting surfaces to scattered data. In proceedings of Curves and Surfaces in Computer Vision and Graphics 3 (SPIE proceedings Vol. 1830), pp. 212 $220,1992$.

\section{Technical Reports}

[29] J.A. McDonald. W. Stuetzle, and A. Buja. Viewing high dimensional data by painting multiple views. Technical Report No. 174, Department of Statistics, University of Washington, 1989.

[30] J.A. McDonald, W. Stuetzle, and A. Buja. Painting multiple views: an interactive technique for "feeling" the shape of complex, multi-dimensional data. Technical Report No. 178, Deptartment of Statistics, University of Washington, 1989.

[31] M. Sarnella. Analyzing Interactive Graphics Systems. Technical Report No. 182, Department of Statistics, University of Washington, 1989.

[32] S. Weghorst and W. Stuetzle. Jeepers: An Interface Perception Research Tool. Technical Report No. 185, Department of Statistics, University of Washington, 1989.

[33] D.D. Cox and F. O'Sullivan, Generalized non-parametric regression. Technical Report, Department of Statistics, University of Washington, 1990.

[34] J.A. McDonald and M. Sannella. Arizona overview and notes for release 0.0. Technical Report No. 202, Department of Statistics, University of Washington, 1991.

[35] J.A. McDonald and M. Sannella. Geometry: a package for basic geometric calculation in Common Lisp, release 0.0. Technical Report No. 203, Department of Statistics, University of Washington, 1991. 
[36] J.A. McDonald and M. Sannella. Slate: a portable bitmap graphics package for Common Lisp, release 0.0. Technical Report No. 204, Department of Statistics, University of Washington, 1991.

[37] J.A. McDonald and M. Sannella. Chart: a simple Common Lisp package for plotting dr.ta and functions, release 0.0. Technical Report no. 205, Department of Statistics, University of Washington, 1991.

[38] T. DeRose, T. Duchamp, H. Hoppe, J.A. McDonald, and W. Stuetzle. Reconstructing two-dimensional manifolds from scattered data: Motivation and background. Technical Report No. 215, Depaprtment of Statistics, University of Washington, 1991.

[39] J.A. McDonald and M. Sannella. Geometry: a package for basic geometric calculation in Common Lisp, release 0.0. Technical ReportNo. 218, Department of Statistics, University of Washington, 1991.

[40] J.A. McDonald. A simple graph browser. Technical Report No. 219, Department of Statistics, University of Washington, 1991.

[41] J.A. McDonald. Definitions: a simple database for typesetting documentation. Technical Report No. 220, Department of Statistics, University of Washington, 1991.

[41] J.A. McDonald and M. Niehaus. Announcements: an implementation of implicit invocation. Technical Report No. 221, Department of Statistics, University of Washington, 1991.

[42] J.A. McDonald. Calling NPSOL from Common Lisp. Technical Report No. 222, Department of Statistics, University of Washington, 1991.

[43] J.A. McDonald and M. Sannella. Arizona overview and notes for release 1.0. Technical Report No. 223, Department of Statistics, University of Washington, 1991 .

[44] T. Duchamp and W. Stuetzle. The geometry of principal curves in the plane Technical Report No. 250, Department of Statistics, University of Washington, 1993.

\section{Video Tapes}

[45] W. Stuetzle. Odds Plots: examining associations between views of a data set. Video tape, 20 minutes; Department of Statistics, University of Washington, 1988.

[46] W. Stuetzle. Analysis of fish abundance in the Bering Sea: a case study in the use of graphical methods. Video tape, 15 minutes; Department of Statistics, University of Washington, 1989. 
[47] A. Buja, J.A. McDonald, J. Michalak, W. Stuetzle, and S. Willis. Visualization of quantitative data. Video tape, 27 minutes; Department of Statistics, University of Washington, 1990.

\section{Submitted}

[48] K. Anderson and F. O'Sullivan. An efficient statistical deconvolution method for episodic hormone data. Biometrics (submitted), 1991.

[49] F. O'Sullivan, Y. Pawitan, and D. Haynor. A comparison of least squares and maximum likelihood image reconstruction in emission computed tomography. IEEE Transactions on Medical Imaging (submitted), 1991.

[50] F. O'Sullivan. Penalized likelihood-type estimation in Pet and more general inverse problems involving linear operators. Annals of Statistics (under revision), 1992. 


\section{Ph.D. Theses}

Work on the following Ph.D. theses was partially or completely supported by the grant:

[51] K. Anderson. Efficient deconvolution of episodic hormone data. Department of Biostatistics, University of Washington, 1991.

[52] J. Michalak. Visualization of tree-based models. Department of Statistics, University of Washington. (Degree awarded posthumously, 1991)

[53] M. Sannella. Solving and debugging constraints with the SkyBlue constraint solver. Department of Computer Science, University of Washington. (Expected to graduate 1993).

[54] C. Huang. Penguin - a system for graphical data analysis. Department of Biostatistics, University of Washington, 1992.

[55] S. McKinney. Automatic and assisted painting. Department of Biostatistics, University of Washington. , Expected to graduate 1993).

[56] H. Hoppe. Surface reconstruction from unorganized points (tentative title). Department of Computer Science, University of Washington. (Expected to graduate 1994). 


\section{Invited Lectures}

\section{Refereed Talks at Conferences}

[1] J.A. McDonald and W. Stuetzle. Data visualization using focusing and linking. Workshop on Size and Complexity in Information Worlds at CHI'91, New Orleans, April 28-29, 1991.

[2] A.Buja, D. Haynor, J.A. McDonald, J. Michalak, Finbarr O'Sullivan, and W. Stuetzle. Graphical tools for quantitative radiology. IEEE 1990 Nuclear Science Symposium: Medical Imaging Conference, October 1990.

[3] T. DeRose, T. Duchamp, H. Hoppe, J.A. McDonald, and W. Stuetzle. Determining surface topology from unorganized data. Second SIAM Conference on Geometric Design, Phoenix, Nov 4-8, 1991.

[4] T. DeRose, T. Duchamp, H. Hoppe, J.A. McDonald, and W. Stuetzle. Fitting a surface of known topology to unorganized data. Second SIAM Conference on Geometric Design, Phoenix, Nov 4-8, 1991.

[5] H. Hoppe, T. DeRose, T. Duchamp, J.A. McDonald, and W. Stuetzle. Surface reconstruction from unorganized points. Sigggraph 1992.

\section{Invited Talks at Conferences}

[6] J.A. McDonald and W. Stuetzle. Analysis of fish abundance in the Bering Sea: a case study in the use of graphical methods. American Statistical Association Annual Meeting, New Orleans, August 22 - 25, 1988.

[7] W. Stuetzle. Statistical graphics. CBMS Research Conference on ComputerIntensive Methods, Washington DC, June 12 - 16, 1989.

[8] W. Stuetzle. Examining associations between views of a data set. IMA conference on Robustness, Diagnostics, Computing, and Graphics in Statistics, Minneapolis, July 10 - September $1,1989$.

[9] W. Stuetzle. A comparison between Lisp and S. IMA conference on Robustness, Diagnostics, Computing, and Graphics in Statistics, Minneapolis, July 10 - September 1, 1989.

[10] J.A. McDonald and J. Pedersen. Geometric abstractions for constrained optimization of layouts. IMA conference on Robustness, Diagnostics, Computing, and Graphics in Statistics, Minneapolis, July 10 - September 1, 1989.

[11] J.A. McDonald. Geometric abstractions in scientific computing. IBM Scientific Research Lab, Palo Alto, CA., October 1989. 
[12] J.A. McDonald and J. Pedersen. A case study of statistical computing in a Lisp environment: geometric abstractions for optimization, graph layout, browsing and retrieval in large databases. American Statistical Association Annual Meeting, 1989.

[13] F. O'Sullivan. Multidimensional smoothing methods: direct and indirect. American Statistical Association Annual Meeting, 1989.

[14] F. O'Sullivan. Sensitivity analysis for system identification. SIAM Special Meeting on Optimization, Boston, 1989.

[15] J.A. McDonald. Geometric abstractions in scientific computing. Computer Science and Statistics, the 22th Symposium on the Interface, East Lansing, Michigan, May 1990.

[16] A. Buja, J.A. McDonald, and W. Stuetzle. Painting multiple views: an interactive technique for "feeling" the shape of complex, multi-dimensional data. Invited Poster, Conference on Human Factors in Computing Systems, Seattle, April 1-5, 1990.

[17] S. Weghorst and W. Stuetzle. Jeepers: an interface perception research tool. Invited Poster, Conference on Human Factors in Computing Systems, Seattle, April 1-5, 1990.

[18] J.A. McDonald, J. Michalak, and W. Stuetzle. Interactive graphics for tree-based models. Biometrics Society ENAR Spring Meeting, Baltimore, April 1-4, 1990.

[19] W. Stuetzle. Analysis of fish abundance in the Bering Sea - a case study in the use of graphical methods. IMS/WNAR Western Regional Meetings, Bozeman, June 17-20, 1990.

[20] J.A. McDonald, J. Michalak, and W. Stuetzle. Interactive graphics for tree-based models. American Statistical Association Annual Meeting, Anaheim, August 5 - 9, 1990.

[21] F. O'Sullivan. Smooth mixture estimation with multichannel image data. 23'rd Symposium on the Interface of Computer Science and Statistics, 1990. [22] F. O'Sullivan, Y. Pawitan, R.L. Harrison, and T.K. Lewellen. PET reconstruction: improving least squares solutions. IEEE 1990 Nuclear Science Symposium: Medical Imaging Conference, 1990.

[23] J.A. McDonald, J. Michalak, and W. Stuetzle. Focusing and linking as paradigms for the visualization of high-dimensional data and other complex objects. SoftStat ' 91 Conference on the Scientific Use of Statistical Software, Heidelberg, April $\mathbf{\imath}-12,1991$. 
[24] W. Stuetzle. Principal curves. Conference on Total Least Squares, Louvain, August 12-16, 1991.

[25] J.A. McDonald, J. Michalak, and W. Stuetzle. Focusing and linking as paradigms for the visualization of high-dimensional data and other complex objects. Seminar on Scientific Visualization, Schloss Dagstuhl, August 2630, 1991.

[26] F. O'Sullivan. Multivariate methods in the analysis of dynamic emission tomography data. Workshop on Curve Estimation, Heidelberg 1991.

[27] F. O'Sullivan. Metabolic Image from Dynamic Emission Tomography Data. American Statistical Association Annual Meeting, Atlanta, 1991.

[28] F. O'Sullivan, Y. Pawitan, and M.M. Graham. Metabolic imaging techniques for dynamic PET data. IEEE 1991 Nuclear Science Symposium: Medical Imaging Conference, 1991.

[29] F. O'Sullivan. Inverting the attenuated Radon transform. Workshop on Empirical Processes and Statistics, Oberwolfach, 1991.

[30] H. Hoppe, T. DeRose, T. Duchamp, J.A. McDonald, and W. Stuetzle. Reconstruction of two-dimensional manifolds from scattered data. Annual Meeting of the German Classification Society, Dortmund, April 1992.

[31] H. Hoppe, T. DeRose, T. Duchamp, J.A. McDonald, and W. Stuetzle. Fitting surfaces to scattered data. sCIE's OE/Technology '92, Boston, November 15-20, 1992. 

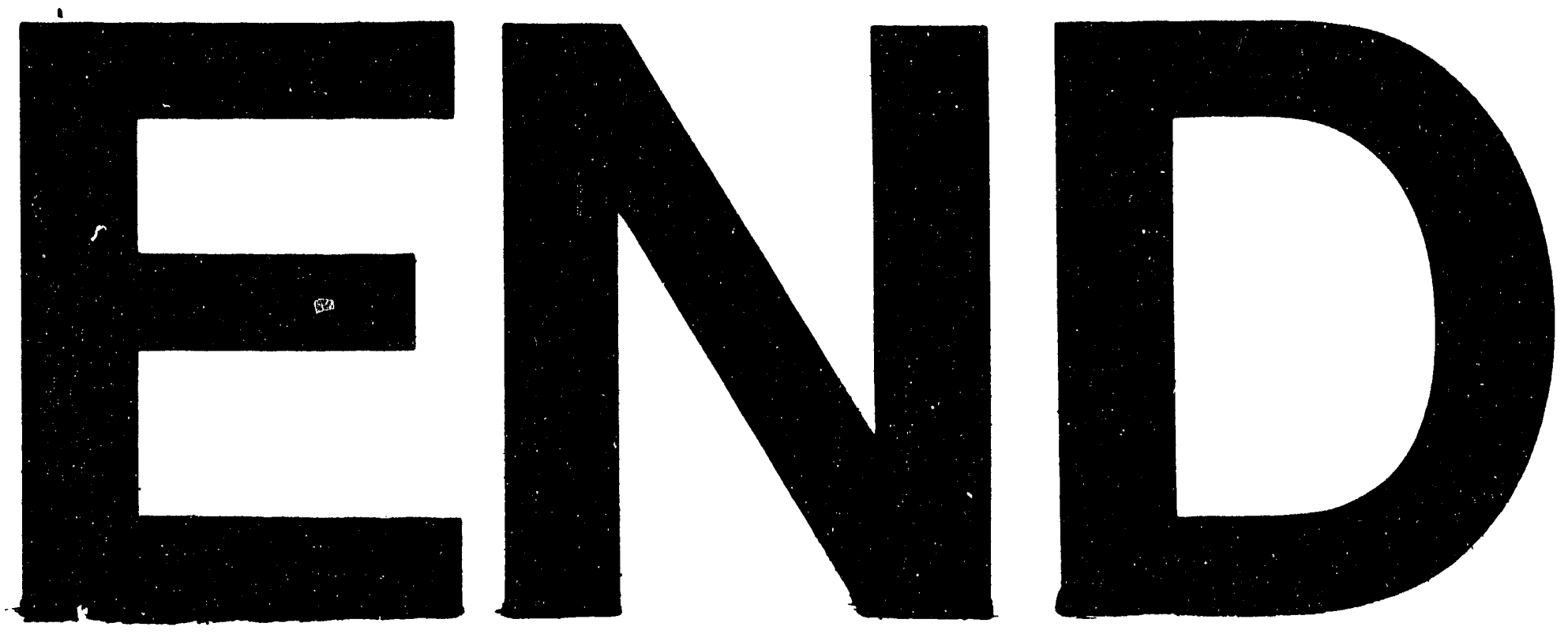

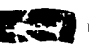

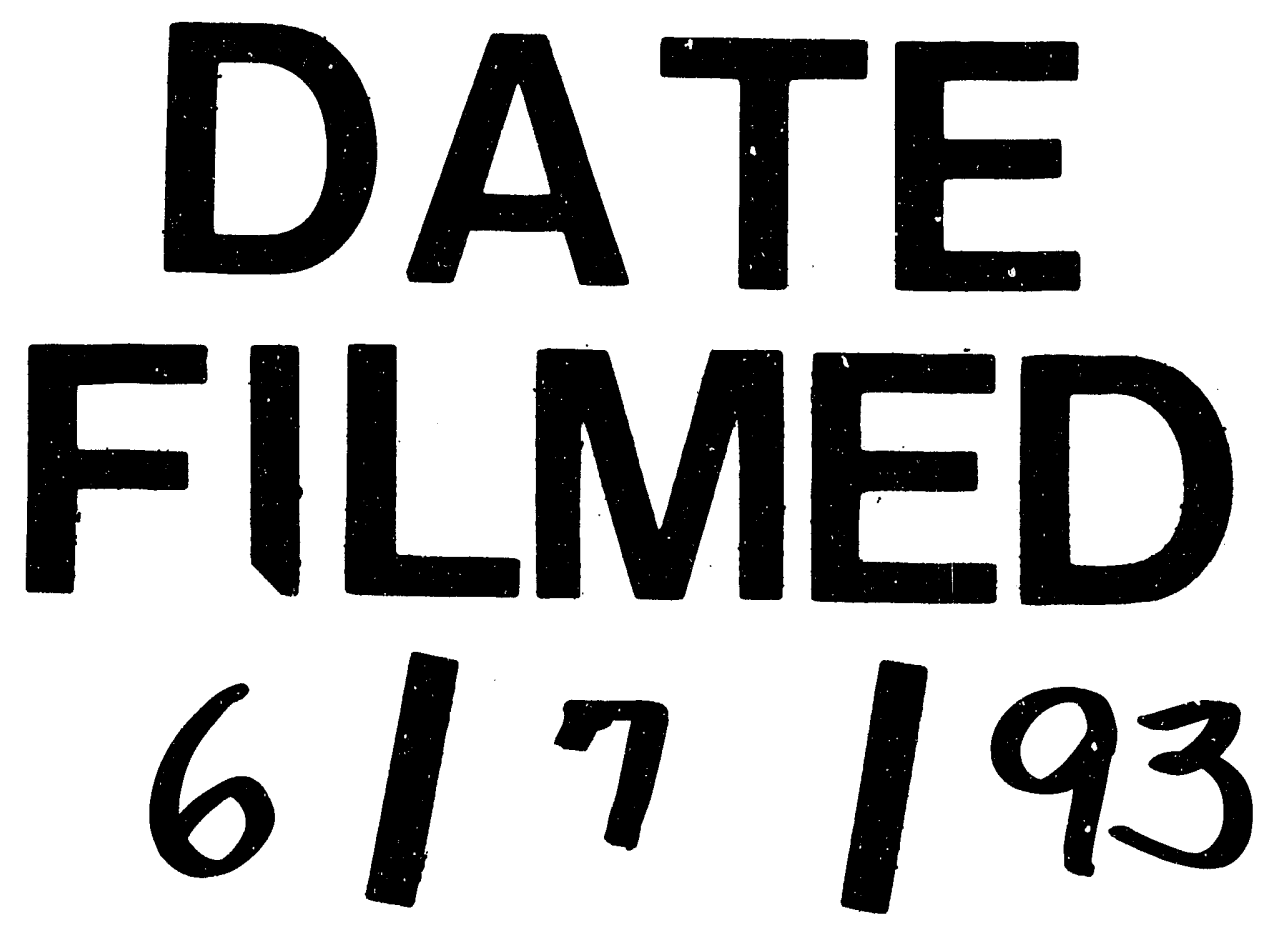

